\title{
Correction to: An Energy Efficient Protocol to Mitigate Hot Spot Problem Using Unequal Clustering in WSN
}

\section{Sunil Kumar Singh ${ }^{1} \cdot$ Prabhat Kumar $^{1} \cdot$ Jyoti Prakash Singh ${ }^{1}$}

Published online: 13 August 2018

(C) Springer Science+Business Media, LLC, part of Springer Nature 2018

\section{Correction to: Wireless Pers Commun (2018) 101:799-827 https://doi.org/10.1007/s11277-018-5716-3}

The Acknowledgement section was missing in the original publication. It is provided below.

Acknowledgements The authors would like to acknowledge the Ministry of Electronics \& Information Technology (MeitY), Government of India, for providing financial assistance for this research work through "Visvesvaraya PhD Scheme for Electronics \& IT".

The original article can be found online at https://doi.org/10.1007/s11277-018-5716-3.

Sunil Kumar Singh sunil.cse14@nitp.ac.in

Prabhat Kumar prabhat@nitp.ac.in Jyoti Prakash Singh jps@nitp.ac.in

1 Department of Computer Science and Engineering, National Institute of Technology Patna, Patna, Bihar, India 